\title{
Comparing the Growth Performance of Baby Chicks under Hay- Box Brooder Technology and Infra-Red Electric Brooder
}

\author{
Merwan Ahmedsham, Kedir Tase, Mulugeta Yoseph, Mahmed Aliyi, Buzinesh Adera, Metekia \\ Tamiru*, Wassihun Hasen \\ Jimma University College of Agriculture and Veterinary Medicine Department of Animal Science, Ethiopia \\ *Corresponding Author: Metekia Tamiru, Lecturer, Department of Animal Science, College of \\ Agriculture and Veterinary Medicine, Jimma University, Ethiopia. Email: metekiatam@gmail.com
}

\begin{abstract}
In Ethiopia, chicks are raised by the broody hen, the success of which depends on the prevalence of predators. Using the artificial brooder technology the benefit from chicken production can be increased. The brooder is used to imitate the warmth and protection like a hen gives to chicks during the brooding stage. Hay Box Brooder (HBB) and Infra-Red Electric Brooder (IRR) were the common methods successfully used to raise layer type chicks. In this experimental study, a total of 180 two day old chickens, of which 90 were kept under HBB and the rest were in IRR to compare the effect of the hay-box brooder and infra-red electric brooder in the growth performance of baby chicks in terms of weight gain. The weight gain of chicks was measured each week in the whole five-week of the brooding period and presented as mean \pm standard error of the mean (SEM) at the significant level of $P<0.05$. In the first week (WI), the weight gained from each brood type was relatively similar $(H B B=38.79 \pm 0.58, I R R=38.05 \pm 0.61)$ respectively. However, after week one onwards, the weight gaining performance of chicken in IRR brooder in W2 (63.73 \pm 1.23$)$, W3 (109.47 \pm 1.85$), W 4(143.22 \pm 3.26)$ and W5 (211.80 \pm 4.02$)$ which were significantly higher than that of the chickens kept in $H B B(W 2=51.39 \pm 0.96, W 3=83.22 \pm 1.86, W 4=130.39 \pm 2.61, W 5=174.68 \pm 3.36)$. The average daily weight gain was higher in the IRR brooder than HBB. This can be explained as HBB didn't allow night feeding and there was stress due to crowdedness as the chicks grow and increase their body size. In addition, the chickens in both brooders were categorized into black, brown and white due to the color they possessed although were the same breed. But in most cases, the black colored showed significantly better growth performance than the white due to unknown reason. In conclusion, all breed in IRR gained higher body weight than those kept under HBB. So that infra-red electric brooder was found to be better for chicken brooding and it should be used widely while hay box brooding should also be used in rural areas where electricity is limited.
\end{abstract}

Keywords: Chick brooding, Growth performance, Hay Box Brooder, Infra-Red Electric Brooder.

\section{INTRODUCTION}

Chick brooding refers to the early periods of growth (0 to 8 weeks) when young chicks are unable to maintain their normal body temperature without the aid of supplementary heat. It is by natural brooding that day-old chicks are raised all over rural Ethiopia. The broody hen rearing and protecting few chicks ceases laying the egg during the entire incubation and brooding periods of up to 81 days (Getinet et al., 2013).

Due to lack of temperature regulation, about 60 $\%$ of the chicks hatched in the countryside area of Ethiopia die during the first eight weeks of age (Tadele and Ogle, 2001) as day-old chicks need external heating to regulate own body temperature. The brooder is used to imitate the warmth and protection like a hen gives to chicks

ARC Journal of Animal and Veterinary Sciences during the brooding stage. The productivity of village chicken production systems in general and the free-range system, in particular, is low (Kondombo, 2005). This is due to low egg production and high mortality rate (Nigussie et al. 2003).

Aberra (2000) also characterized the low productivity of local chicken due to low egg production performance, production of smallsized eggs, slow growth rate, late maturity, small clutch size, an instinctive inclination to broodiness and high mortality of chicks. Yet the success of the brooding process depends on the maternal instinct of the broody hen and the prevalence of predators such as birds of prey, pets, and some wild animals, all of which are listed as the major causes of premature death of chicks in Ethiopia (Getinet et al., 2013). 
The hay-box chick brooder, developed at Jimma University College of Agriculture and Veterinary Medicine (JUCAVM) is an appropriate and simple technique that could be used to raise day-old chicks at the village level. The technology utilizes simple and locally available materials and involves brooding of chicks by conserving their own metabolic heat to keep them warm. Under village conditions, the use of hay-box brooder has the advantages of providing protection against predator attack and reduces the risk of exposure to disease through confinement (Nigussie et al., 2003).

The hay- box chick brooder was comprehensively studied using layer type dayold chicks in Ethiopia and found to be the brooders of choice for the promotion of day-old chick's poultry extension package under the Ethiopian household farming conditions, hence it has been proven to increase the growth performance of broiler chicken (Getinet et al., 2013). Mean mortality level of 18.7 and $19.6 \%$ were recorded from groups of layer type day-old chicks assigned to the electric and hay-box brooder respectively (Nigussie et al., 2003). Unfortunately, however, there was adequate research conducted to evaluate the suitability of hay-box brooder in raising Layers and broilers of types of two-day-old chicks in the study areas under the existing environmental conditions. Therefore, the objective of the study was to compare the effect of hay box brooder and infrared electric brooder in the growth performance of two-day-old baby chicks.

\section{Materials AND Methods}

\subsection{Study Area}

An experimental study was conducted between April 2016 to June 2016 at Brooding and Incubation house of poultry farm of Jimma University College of Agriculture and Veterinary Medicine (JUCAVM), Jimma town. Jimma is located in South West part of Ethiopia $352 \mathrm{~km}$ far from Addis Ababa at latitude of about $7^{\circ} 13^{\prime}-8^{\circ} 56^{\prime} \mathrm{N}$ and longitude of about $35^{\circ} 52^{\prime}-37^{\circ} 37^{\prime} \mathrm{E}$ and an elevation ranging from $880 \mathrm{~m}$ to $3360 \mathrm{~m}$ above sea level, but the study site (JUCAVM) has an altitude of 1710 m.a.s.l. The study area receives a mean annual rainfall of about $1530 \mathrm{~mm}$. The annual mean minimum and the maximum temperature is $14.4^{\circ} \mathrm{C}$ and $26.7^{\circ} \mathrm{C}$ respectively.

\subsection{Data Collection and Management of Experimental Chicks}

About 90 randomly selected and weighted two old chicks were transferred to 3 hay box brooder which accommodates 30 chicks with three replications. All of those hay-box brooders were constructed to measure $30 \times 45 \times 45 \mathrm{~cm}$ with the daytime running area of $30 \times 98 \times 98 \mathrm{~cm}$ and each of the constructed hay-box brooders consisted of 4-outer framing boards, each of which is $2 \mathrm{~cm}$ thick. Four small ventilation holes of $2.5 \mathrm{~cm}$ diameter were drilled at the upper side of each frame board (total of 16) and a door of appropriate dimension was fitted at the center of one of the four frame boards.

The other 90 two day old chicks were randomly selected and weighed and kept in three replicate to the infrared electric brooder. Chicks in both treatments were offered commercial starter ration and water for the last five weeks. The hay box grouped chicks were gently pushed out of the box every morning and spent feeding and watering in the run during the daytime and returned back into the box during the night time. Finally, the data on weight gain of chicks were recorded through frequent visits at weekly interval.

\subsection{Data Analysis}

Data were analyzed by one-way analysis of variance (ANOVA) using JMP 12 software (SAS instate Carve, NC USA, 2014) difference between mean were tested using the Tukey Kramer test. The difference was considered significant at $\mathrm{P}<$ or -0.05 . Data are presented as the mean \pm standard error of the mean (SEM). The result was presented in the form of graphs and table.

\section{RESULT AND DISCUSSION}

\subsection{Weight Gaining Performance of Chickens under HBB and IRR}

The two-day-old chickens were separately entered into hay box brooder (HBB) and infrared brooder (IRR) to assess the performance of brooder in weight gaining of chicken. Three hay boxes have been used, each can accommodate 30 chickens, and the other 90 chickens were brooded under infra-red electric brooder. The weight gaining performance of chickens had been recorded during the period of brooding for five weeks.

Those chickens that brooded under the IRR had high weight gaining performance at the end of the brooding period of five-week (211.80 \pm 4.02) while those under HBB had (174.68 \pm 
3.36), this result is similar with the finding of Solomon (2007). According to the data presented in Figure 1, the mean body weight recorded at first time (two days old chick), which was labeled as W1, has no any significant difference (relatively equal weight chicken) for chickens in both brooder, but slight significant difference was indicated during W2, W3, W4, and the significantly higher difference was recorded during the fifth week of brooding (W5) in chickens under infra-red electric brooder at significant level of $(\mathrm{P}<0.01)$. This finding agrees with the report by Matiwos and Selamawit (2014) as BWG of (43.72 \pm 3.87$)$ in 30 chicken accommodating $\mathrm{HBB}$ within five weeks of the brooding period.

The slower growth rate of the box grouped baby chicks was attributed to the fact that the box groups were fed to appetite during day times only and closed at the night as compared to the electric groups, which were fed to appetite both day and night. In W5 of the brooding period, the BWG in IRR has highly increased while HBB increased at a decreasing rate as indicated in Fig.1. Similar to this, Solomon (2007) suggested that comparatively faster growth rate was obtained from the hay-box groups after the first four weeks of brooding during which they doubled their body weight. When all the treatment groups were transferred to their respective rearing corners,(after the brooding period), the hay-box groups rapidly compensated the slow growth they experienced at the early phase of brooding and acclimatized quickly than the electric groups.

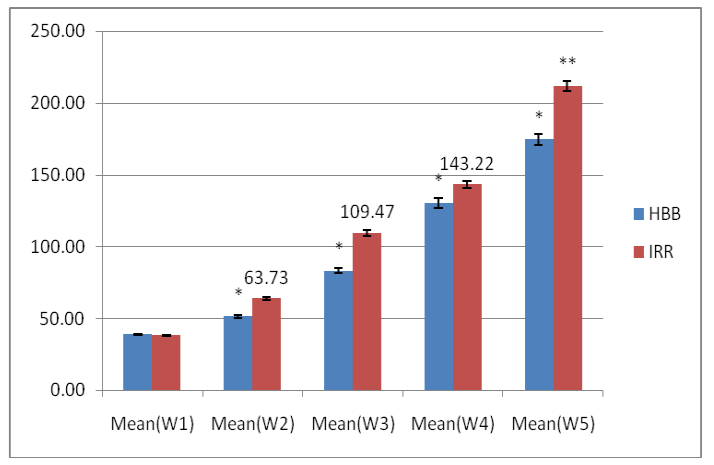

Figure1. The graph indicating comparisons of weight gaining performance of chicken under hay box brooder $(H B B)$ and infra-red brooder (IRR)

\subsection{Weight Gaining Performance of hybrid koekoek chickens (Black color) and Lohmann Brown chickens (Brown or white) under HBB and IRR}

According to this experiment, the weight gaining performance of baby chicks of different breeds had no significant difference in both brooders. Chickens were categorized into three different colors namely as Black, Brown, and White. The koekoek chicken breed was named Black while Lehmann Brown chicken was named White and Brown. The weight gaining performance of these breeds was varying in each week of the brooding period. The weight measured in the first week was the weight of two-day-old chick that had no difference in all breed or color of chickens within the same brooder and/or different brooder.

However, the weight gaining performance in succeeding period of brooding (W2, W3, W4, and W5) was found to be different in chickens under different brooder rather than their color or breed. The higher value of weight gain was obtained in those chickens kept under infra-red brooder than hay box brooder in each week as indicated in Fig. 2, Fig.3, Fig.4 and Fig.5.

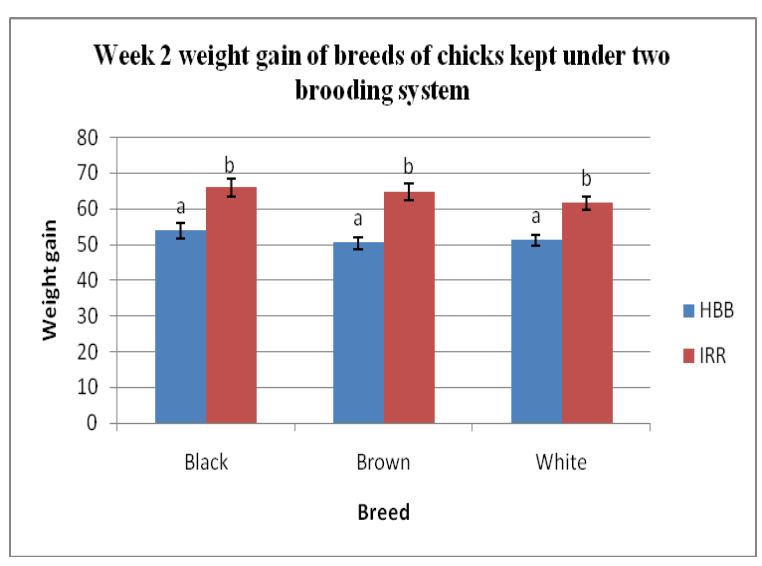

Figure2. Graph indicating Week 2 weight gain of breeds of chicks kept under HBB and IRR brooding system

(The same letter indicates no significant difference; significant differnce between " $a$ " and " $b$ ")

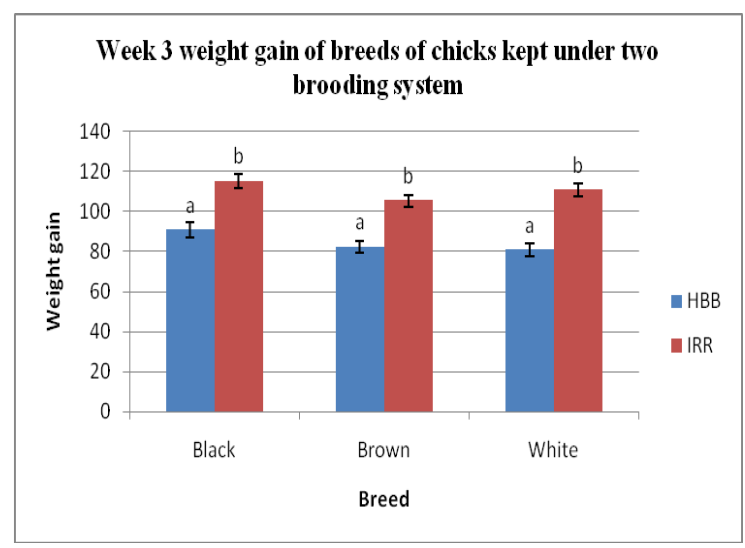

Figure3. Graph indicating Week 3 weight gain of breeds of chicks kept under HBB and IRR brooding system 


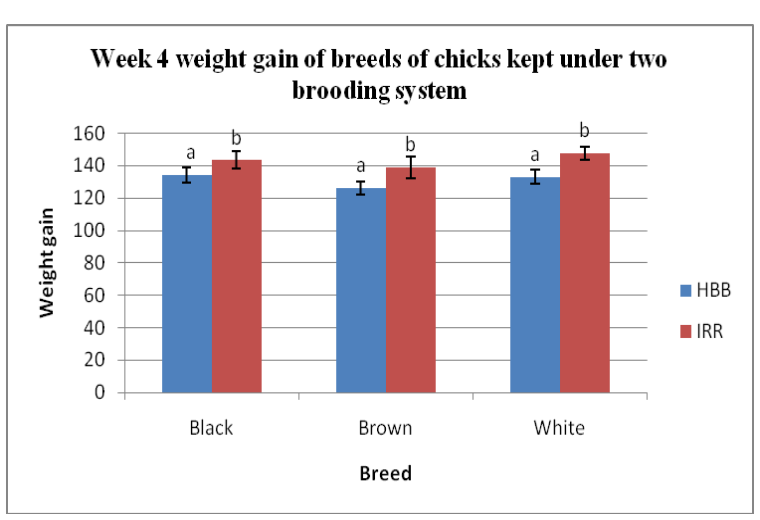

Figure4. Graph indicating Week 4 weight gain of breeds of chicks kept under HBB and IRR brooding system

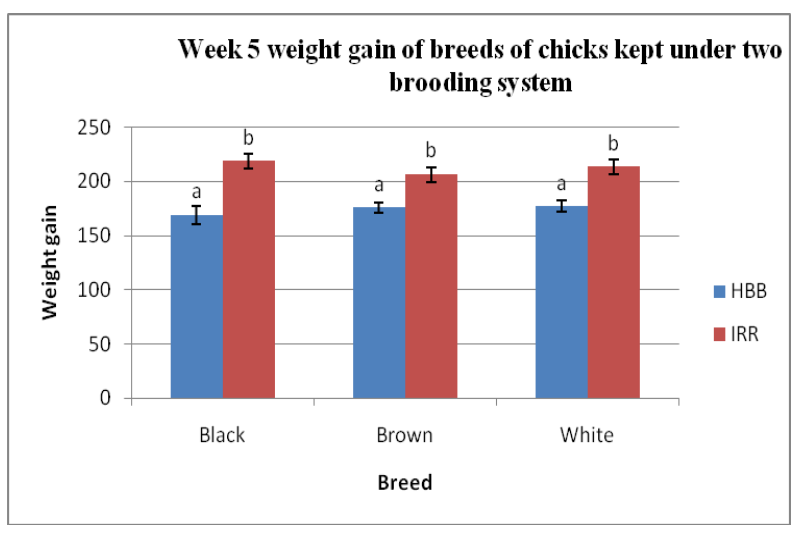

Figure5. Graph indicating Week 5 weight gain of breeds of chicks kept under HBB and IRR brooding system

Importantly, the average daily weight gain was indicated the difference in average daily weight gain between chicken breeds or color those kept under infra-red brooder, but there is no any significant difference in average daily weight gain between chicken breeds or color under hay box brooder. The breeds that were under IRR (black, brown and white) were recorded the significant difference in daily weight gain (31.46842, 25.98485 and 22.13235$)$ respectively as shown on the Fig. 6.

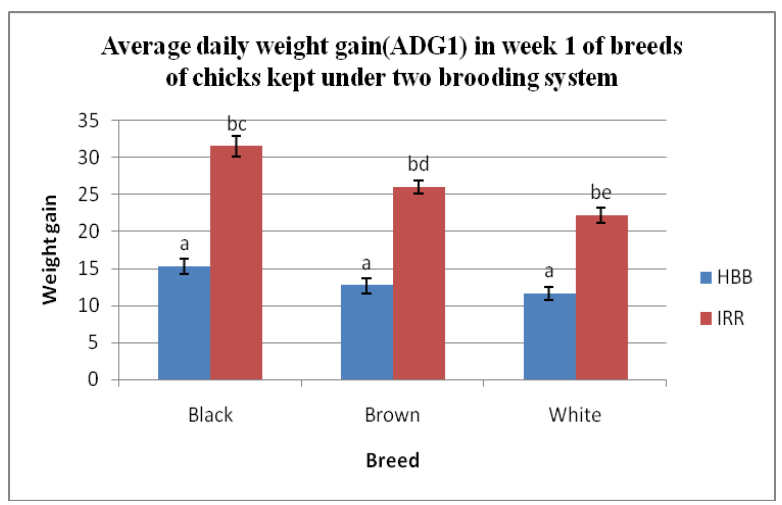

Figure6. Average daily weight gain (ADGl) in week 1 of breeds of chicks kept under HBB and IRR brooding system

ARC Journal of Animal and Veterinary Sciences
However, starting from the scond week of brooding the average daily gain of chickens under hay box brooder bcome vary by a significant range. In that, the average daily weight gain in Black (36.64706), Brown (31.49143) and White (29.13429) breeds (Fig.7) was indicated, but the great significance level of differnce obtained between black and white color or breed. The reason for this difference between the breed in weight gaining is knot known, it needs further research.

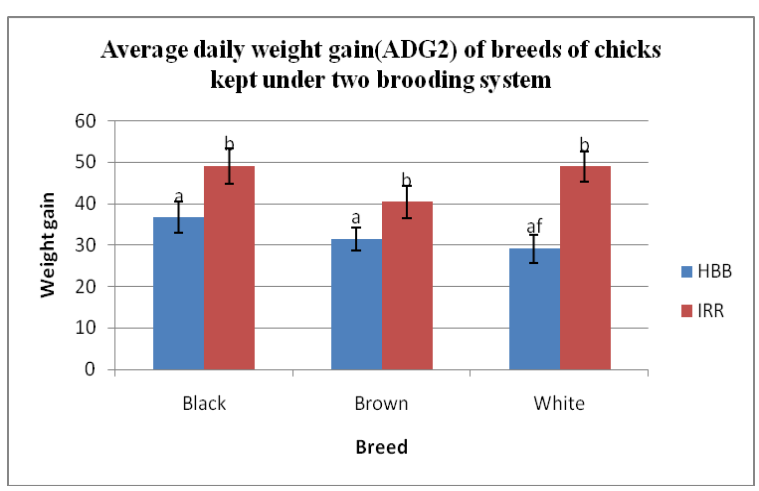

Figure7. Average daily weight gain (ADG2) in week 2 of breeds of chicks kept under HBB and IRR brooding system

Similar to the preceding period of brooding, the average daily weight gain of breeds that was recorded in the third week is relatively similar among those kept under IRR (series 2); this is visualized on Fig.8. However, the great difference was obtained among the black (1), brown (2) and white (3) by their mean \pm standard of error $(28.41053 \pm 7.277747)$, (33.55 $\pm 7.137452)$ and $(36.91176 \pm 4.143278)$ at the significant level of $\mathrm{P}<$ or -0.05 .

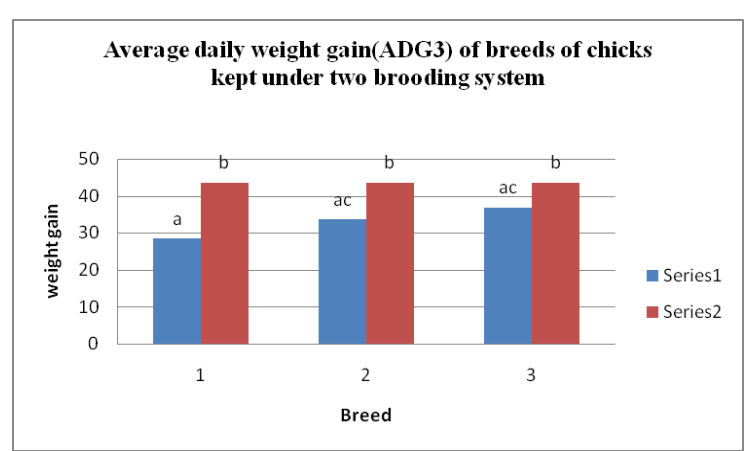

Figure8. Average daily weight gain (ADG3) in week 3 of breeds of chicks kept under HBB and IRR brooding system

In the last week of brooding, the result in average daily weight gain among the breed or color of chicken under both brooders found to be varied. The black breed (34.74706 \pm 11.81827) under $\mathrm{HBB}$ recorded a low value of mean \pm standard of error than brown (46.90286 
$\pm 7.620722)$ and white $(43.81667 \pm 7.520769)$ at a significant level of $\mathrm{P}<$ or -0.05 as indicated in Fig.9. However, the black breed (75.27368 \pm 10.09502) kept under IRR recorded higher value of average daily weight gain in mean \pm standard of error than brown $(63.37097 \pm 8.809698)$ and white $(65.69706 \pm 8.923804)$ at the significant level of $\mathrm{P}<$ or -0.05 .

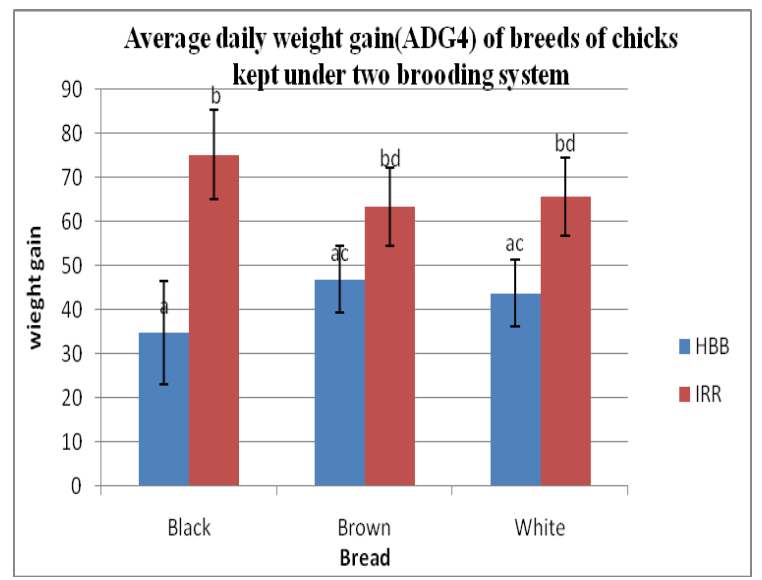

Figure9. Average daily weight gain (ADG4) in week4 of breeds of chicks kept under $H B B$ and IRR brooding system

\section{CONCLUSION AND RECOMMENDATIONS}

This experiment had studied the comparison of growth performance of baby chicks under haybox brooder technology and infra-red electric brooder in order to assess which one is better to provide the higher weight gain of brooding chicken. Chicken brooding is very important which significantly reduce chicken mortality due to temperature, predation etc. Brooding chicks using hay box (HBB) or infra-red brooder (IRR) can alleviate this problem. However, both brooders have different capacity in weight gaining performance of baby chicks. Of the two brooders, infra-red brooder was found to have the efficiency of weight gaining performance of chicken.

During brooding period of five-week (W1 to W5) of this experiment, the weight gained (mean weight \pm standard of error per week) in chicks under infra-red brooder was significantly higher than those chicks kept under hay box brooder. the mean weight gaining performance of chicken in IRR brooder in W2 $(63.73 \pm 1.23)$, W3 $(109.47 \pm 1.85)$, W4 (143.22 \pm 3.26$)$ and W5 $(211.80 \pm 4.02)$ are significantly higher than that of the chickens kept in HBB $(\mathrm{W} 2=51.39 \pm$ $0.96, \mathrm{~W} 3=83.22 \pm 1.86, \mathrm{~W} 4=130.39 \pm 2.61$, $\mathrm{W} 5=174.68 \pm 3.36$ ). IRR allows the brooding chicks to move freely by providing the sufficient temperature and night feeding that reduce stress. Those chickens in the hay box increased their body weight gain at a decreasing rate when compared to IRR, particularly, in w4 and w5. Because the hay box that constructed to accommodate 30 chicks measure $30 \times 45 \times 45$ $\mathrm{cm}$. the space of this box became insufficient as the body size of chicks increased, creating stress of crowdedness (heat) besides deprivation of food during the night.

Also, the average daily weight gain was calculated which expressed the higher value of chicken weight gaining in the IRR brooder than HBB. The chickens in both brooders were categorized into black, brown and white breed or color, but sometimes, the black breed showed the significant difference from white breed for unreasonable factor. However, all breed in IRR gains higher body weight gain than those kept under HBB. So that infra-red electric brooder is better for chicken brooding, but it can't be applicable in the rural area.

Based on the above conclusion the following points are forwarded as the recommendation:

Awareness creation in society about the importance of chicken brooding should be performed.

$>$ Electric supply for society should be increased to enhance the usage of Infra-red brooding technology.

$>$ The number of chicks that allowable to accommodated by a particular hey box size should be reconsidered to alleviate stress due to crowdedness.

\section{REFERENCES}

[1] Abebe, H., 1992. Terminal report on the comparative evaluation of native chicken in the Hararge administrative region and their crosses with the single comb white Leghorn. Mimeographed report. The Alemaya University of Agriculture. pp. 22-27.

[2] Abera, M., 2000 Comparative studies on performance and physiological responses of Ethiopian indigenous (Angete Melata) chickens and their $\mathrm{f} 1$ crosses to long-term heat exposure. $\mathrm{Ph} . \mathrm{D}$. dissertation, Martin-Luther University. Halle-Wittenberg Germany. Pp. 127.

[3] Aklilu H., Almekinders C.J.M., Van der Zijpp and A.J., 2007. Village poultry consumption and marketing in relation to gender, religious 
festivals, and market access. Tropical Animal Health and Production 39, 165-168.

[4] Alamargot, H., 1987. Avian Physiology of Industrial Poultry Farms in Ethiopia. pp. 114117. Proceedings of the First National Livestock Improvement Conference 11-13 Feb. 1987, Addis Ababa, Ethiopia.

[5] Alemu, Y., 1995. Poultry production in Ethiopia. World's Poultry Science Journal.51: 197- 201.

[6] Alemu, Y., and Tadelle D., 1997. The status of poultry research and development in Ethiopia, research bulletin No.4, poultry commodity research program Debrezeit Agricultural research center. Alemaya University of Agriculture, Ethiopia. pp. 62.

[7] Amanda B 2007. General brooding and rearing fact sheet. Introduction to Poultry Production in Saskatchewan (http://www.agriculture.gov.sk.ca)

[8] Australian Agricultural Consulting and Management Company (AACMC), 1984. Livestock sub sector review, Volume 1.

[9] Brannang, E. and Pearson S., 1990. Ethiopian animal husbandry, Uppsala, Sweden, 127pp. Breeding in the Tropics and Sub-tropics, Humboldt University of Berlin, Germany.

[10] Bush, Jennifer, 2006. The Threat of Avian Flu Predicted Impacts on Rural Livelihoods in Southern Nation, Nationalities and Peoples Region (SNNPR), Ethiopia. The Food Economy Group, May 2006.

[11] Central Statistical Authority, 2005. Agricultural sample survey vol. ii; statistical bulletin no. 331, Addis Ababa, Ethiopia.

[12] Demeke, S. (2007). Suitability of hay-box brooding technology to the rural household poultry production system. Livestock research for rural development, 19.

[13] EARO (Ethiopian Agricultural Research Organization), 1999. National poultry research program: Strategy document, Addis Ababa, Ethiopia.

[14] ESAP, 2003. Hay box chick brooding technology hailed a success. Ethiopian Society of Animal Production, Newsletter Issue No 9. Addis Ababa, Ethiopian. Ethiopia. Tropical Animal Health and Production, 33(6): 521-537.

[15] Getinet, A., Solomon, D. and Tadelle, D. (2013). Feasibility of household broiler production with the use of hay box brooder in Ethiopia. International Journal of Livestock Production, 4(5): 70-77

[16] Halima, H., 2007. Phenotypic and Genetic Characterization of indigenous chicken populations in northwest Ethiopia. Ph.D. thesis submitted to the Faculty of Natural and Agricultural Sciences Department of Animal,
Wildlife and Grassland Sciences University of the Free State, Bloemfontein, South Africa.

[17] Kidane, H., 1980. Performance of F1 crossbreeds. Welaita Agricultural Development Unit. Animal husbandry and breeding. Welaita Sodo, Ethiopia. Bulletin No. 4:33p.

[18] Kondombo SR. 2005. Improvement of village chicken production in a mixed (chicken-ram) farming system in Burkina Faso. Ph.D. thesis. Wageningen Institute of Animal Sciences, Animal Nutrition Group, Wageningen University, The Netherlands. pp 208.

[19] Matiwos Habte, Selamawit Debele. On-station and on-farm comparative performance evaluation of hay box chick brooder at Dilla University and Amaro Woreda, Snnprs of Ethiopia. American Journal of Research Communication, 2014, 2(8): 172-188

[20] Mekonnen, G., Teketel F. and Anteneh A., 1991. The Ethiopian Livestock Industry: Retrospect and prospects. Proc.3rd National Livestock Improvement Conference, Institute of Agricultural Research, Addis Ababa, Ethiopia.

[21] Nigussie D, Alemu Y, Tadelle D, Samuel WH (2003). On-station and on-farm evaluation of the 'Hay-Box chick brooder using different insulation Materials at Debre Zeit agricultural research center and Denbi village, Adaa Woreda.

[22] Roberts, J.A. and Gunaratne, S.P., 1992. The scavenging feed resource base for village chickens in a developing country. In Proceedings, 19th World Poultry Congress, Amsterdam, the Netherlands. 20-24 Sep. 1992, Vol. 1, pp. 822-825.

[23] Solomon Demeke, 2001. Suitability of homemade hay box chick brooder to the Jimma and Bedele areas of Oromia Region. Ethiopian. J.Agri.Sci.

[24] Solomon Demeke, 2003. Hay-box chick brooding technology: construction, operation, and management Manuel. Jimma College of Agriculture. Jimma, Ethiopia

[25] Solomon Demeke, 2007: Suitability of hay-box brooding technology to the rural household poultry production system. Livestock Research for Rural Development. Volume 19, Article \#3. Retrieved June 11, 2016, from http://www.lrrd. org/lrrd19/1/deme19003.htm

[26] Solomon Demeke, 2010. Chick Brooder. News article of Spore, CTA. Bimonthly bulletin No. 145 .

[27] Solomon Demeke, 1999. Suitability of homemade hay-box chick brooder to the Ethiopian household poultry production system. 
Proceedings of the 7th annual Conference of the Ethiopian Society of Animal Production. Addis Ababa, Ethiopia

[28] Solomon Demeke. 2012. Hay Box Brooder: A Milestone to Increase Rural Households Poultry Production. INFPD Good Practices of Family Poultry Production Note No 01.
[29] Spore (2010). Chick Brooder. News article of Spore, CTA. Bimonthly bulletin No.145. The Netherlands. spore.cta.int/index.

[30] Tadele, D. and Ogle, B. (2001). Village poultry production systems in the central highlands of

[31] Teketel, F., 1986. Studies on the meat production potentials of some local strains of chickens in Ethiopia. Ph.D. thesis presented to the University Giessen, Germany, pp123-126.

Citation: Merwan Ahmedsham, Kedir Tase, Mulugeta Yoseph, Mahmed Aliyi, Buzinesh Adera, Metekia Tamiru et al. Comparing the Growth Performance of Baby Chicks under Hay-Box Brooder Technology and Infra-Red Electric Brooder. ARC Journal of Animal and Veterinary Sciences. 2018; 4(1):26-33. doi: dx.doi.org/ 10.20431/2455-2518.0401005.

Copyright: (c) 2018 Authors. This is an open-access article distributed under the terms of the Creative Commons Attribution License, which permits unrestricted use, distribution, and reproduction in any medium, provided the original author and source are credited. 